\section{Deutliche Unterschiede bei Mucilaginosa}

— Bei Heiserkeit und Halskratzen sind Hyaluronsäure haltige Präparate eine Therapieoption. In einer Vergleichsstudie mit drei anderen Präparaten zeigte sich GeloRevoice ${ }^{\circledast}$ deutlich überlegen. In die prospektive Beobachtungsstudie wurden 80 Patienten mit akuten Hals- und Stimmproblemen im Alter von 18-90 Jahren aufgenommen. Bakterielle Infektionen wurden ausgeschlossen. Die Patienten erhielten entweder GeloRevoice ${ }^{\circledR}$ oder eins von drei anderen Mucilaginosa. Es erfolgte eine Bewertung der Symptome durch Arzt und Patient anhand eines Scores (1-10 Punkte) zu Beginn und am Ende der Therapie (im Mittel fünf Tage).

Die Ergebnisse: Lag das Beschwerdeausmaß zu Beginn zwischen 3,5 und 4,3 Punkten, verringerte es sich in allen vier Gruppen, berichtete Prof. Hans Behrbohm, Park-Klinik Weißensee, Berlin. Bei den Vergleichs-präparaten lag der Wert zwischen 1,9 und 2,1 Punkten, unter GeloRevoice ${ }^{\circledast}$ bei nur 0,9 (Ausgangswert: 4,3). „Die Reduktion aller Symptome gelang mit dem Testpräparat bei fast $80 \%$ der Patienten", so der HNOFacharzt. „Bei den Vergleichspräparaten war das nur bei rund 40 bis $48 \%$ der Patienten der Fall." Unter dem Testpräparat waren zudem an Tag drei $40 \%$ Patienten beschwerdefrei, mit den Vergleichspräparaten nur rund $10-15 \%$. Behrbohm führt das auf die lange Verweildauer des Schutzfilms durch das Hydro-Depot ${ }^{\circledR}$ zurück. So seien die Schleimhäute besser und länger geschützt. "GeloRevoice ${ }^{\circledast}$ ist anderen Mucilaginosa deutlich überlegen", fasste Behrbohm die Ergebnisse zufassen.

Das Medizinprodukt GeloRevoice ${ }^{\circledR}$ ist für Erwachsene und Kinder ab sechs Jahren zugelassen. Es ist in drei Geschmacksrichtungen erhältlich (Kirsch-Menthol, CassisMenthol, Grapefruit-Menthol). Bis zu sechs Tabletten täglich können gelutscht werden, vorzugsweise nach dem Essen. „Das verhindert die Beschädigung des Schutzfilms durch Nahrungsbestandteile", so Behrbohm.

Dr. Michael Hubert

Medien-Event „GeloRevoice: Besser gut bei

Stimme. Lass Deine Stimme klingen wie

10.000!", Hamburg, 4. August 2016

Veranstalter: Pohl-Boskamp

\section{Hörtraining per Smartphone für Hörimplantat- Träger}

— Nutzer mit einem Cochleaimplantat $(\mathrm{Cl})$ müssen das Hören wieder neu lernen. Erst mit regelmäßigem Training ist das Gehirn in der Lage, Höreindrücke als Sprache, Musik oder Blätterrauschen zu interpretieren. Bislang konnten Cl-Träger mit ihrem Therapeuten, mit Freunden, Bekannten, Familienmitgliedern oder alleine mit einer der „Listen Up!“-CDs üben. Dadurch war die Flexibilität des Trainings aber oftmals eingeschränkt. MED-EL erweitert seine bewährte "Listen-Up!"-Trainingsreihe nun um eine Smartphone-App, die CochleaimplantatTrägern vielfältige Übungsmöglichkeiten bietet. Nutzer können ihr Gehör ab sofort flexibel und jederzeit trainieren - die neue Android-App lässt sich als Ergänzung zu den „Listen-Up!”Trainings-CDs nutzen. Besondere Technik-Kenntnisse sind nicht notwendig: Die App ist übersichtlich aufgebaut, einfach zu bedienen und bietet die Möglichkeit, das Training den persönlichen Anforderungen anzupassen. Die Aufgaben sind in verschiedene Schwierigkeitsstufen unterteilt - ein separater Test hilft, den eigenen Trainingserfolg immer wieder zu überprüfen. Der Anwender hat die Möglichkeit, ein individuelles Trainingsprofil zu erstellen, etwa zwischen einer männlichen oder weiblichen Stimme für die Darbietung der Aufgaben zu wählen, Hintergrundgeräusche zuzuschalten oder den Aufgabentext ein- oder auszublenden. Die App trainiert gezielt das Verstehen von Wörtern, Sätzen und ganzen Texten. Durch den stufenweisen Übungsaufbau können sich die Nutzer in ihrem eigenen Tempo Schritt für Schritt verbessern. Ab sofort sind die einzelnen Trainingsstufen für 2,49€ im Google Play Store erhältlich (Komplettpaket mit zehn Stufen inkl. Test kostet 24,99€). Wer nur den Test absolvieren möchte, kann diesen separat für $3,99 €$ herunterladen.

red

Nach Informationen von Med El

\section{Asthma und COPD: Erfolgreiche Therapie ist auch eine Frage der Technik}

— In Deutschland leiden 9,9\% der Erwachsenen unter Asthma und 8,6 \% unter COPD. Zur Behandlung stehen verschiedene medikamentöse Optionen zur Verfügung, wobei nationale und internationale Leitlinien vorrangig die inhalative Applikation der Medikamente empfehlen. Doch bis zu $80 \%$ der Patienten begehen mindestens einen Fehler bei der Anwendung ihres Inhalationssystems. Eine der Folgen beschrieb Privatdozent Dr. Kai-Michael Beeh, Wiesbaden, auf dem Teva-Industriesymposium „Device matters - Looking beyond the drug" am 4. September 2016 anlässlich des diesjährigen Kongresses der European Respiratory Society (ERS) in London: „Wenn durch die Fehlanwendung des Inhalators keine ausreichende Wirkung erzielt wird, resultiert daraus häufig ein unkontrolliertes Asthma. Für den Arzt ist es dann im Einzelfall schwierig zu erkennen, ob die resultierende Symptomatik Ausdruck des Asthma-Schweregrads ist oder auf inkorrekter Inhalation beruht." Die richtige Anwendung des Inhalators sowie eine korrekte Schulung sind von großer Bedeutung.

Einfach anzuwendende Behandlungsoptionen wie der DuoResp ${ }^{\circledR}$ Spiromax ${ }^{\circledR}$ erleichtern die Therapie von Asthma- und COPD-Patienten und können viele Anwendungsfehler vermeiden helfen oder beinträchtigen nicht die Wirkstofffreisetzung. Der DuoResp ${ }^{\circledast}$ Spiromax ${ }^{\circledast}$ enthält die bewährte Fixkombination aus Budesonid und Formoterol und funktioniert nach dem intuitiven Prinzip „Öffnen - Inhalieren - Schließen“. Die spezielle Technik im Inneren gewährleistet, dass lageunabhängig und schon bei vergleichsweise geringen Inspirationsflüssen die vorgesehene Dosis präzise abgegeben wird.

red

Nach Informationen von TEVA 